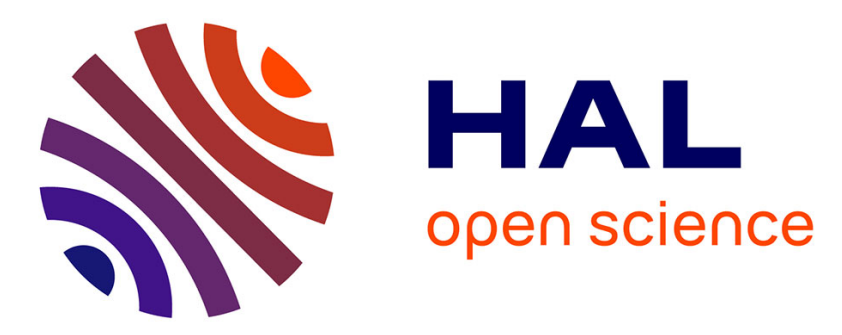

\title{
Le substrat africain dans la résistance des rois marrons Bayano (Panama, 1554) et Yanga (Mexique, 1609) Jean-Pierre Tardieu
}

\section{To cite this version:}

Jean-Pierre Tardieu. Le substrat africain dans la résistance des rois marrons Bayano (Panama, 1554) et Yanga (Mexique, 1609). Journal des Africanistes, 2016, 86 (2), pp.122-139. hal-01531466

\author{
HAL Id: hal-01531466 \\ https://hal.science/hal-01531466
}

Submitted on 31 Oct 2018

HAL is a multi-disciplinary open access archive for the deposit and dissemination of scientific research documents, whether they are published or not. The documents may come from teaching and research institutions in France or abroad, or from public or private research centers.
L'archive ouverte pluridisciplinaire HAL, est destinée au dépôt et à la diffusion de documents scientifiques de niveau recherche, publiés ou non, émanant des établissements d'enseignement et de recherche français ou étrangers, des laboratoires publics ou privés. 


\title{
Le substrat africain dans la résistance des rois marrons Bayano (Panama, 1554) et Yanga (Mexique, 1609)
}

Jean-Pierre Tardieu

Université de La Réunion

\section{Résumé}

La résistance des esclaves marrons a fortement marqué l'histoire des Amériques espagnoles depuis les débuts de la colonisation jusqu'aux indépendances. A cet égard, les cas des communautés de Bayano au Panama en 1554 et de Yanga au Mexique en 1609 furent très significatifs. Les responsables administratifs et religieux virent dans leur comportement une manifestation de barbarie, alors que, en particulier dans les domaines de l'organisation sociale, de la religion et de la guerre, il trouvait sa justification dans un authentique substrat africain, que ne parvenait pas à effacer la tentative d'acculturation chrétienne.

Mots clés: Esclaves marrons. Bayano. Panama. 1554. Yanga. Mexique. 1609. Substrat africain

\begin{abstract}
The resistance of maroon slaves left its marks in the Spanish America history from the beginnings of the colonization through to the Independence. In that respect, the communities of Bayano (Panama, 1554) and Yanga (Mexico, 1609) were very significant. Administrative and religious leaders saw their behaviour as a display of barbarism, particularly in the areas of social organization, religion and war. However, this behaviour had a justification rooted in an authentic African substratum, which attempts at Christian acculturation had not managed to obliterate.
\end{abstract}

Keywords : Maroon slaves. Bayano. Panama. 1554. Yanga. Mexique. 1609. African substratum.

La résistance des esclaves aux Amériques se manifesta très tôt, avec la révolte des Noirs de l'hacienda sucrière de Diego Colón, le fils de Christophe Colomb, à la Noël 1522 dans l'île d'Hispaniola. D'autres soulèvements s'ensuivirent, toujours jugulés, d'où le choix d'une autre stratégie, individuelle cette fois, à savoir la fuite. Le marronnage est presque aussi vieux que l'esclavage aux Amériques. Le mot « cimarrón » est d'ailleurs d'origine arawak (Tardieu 2006 : 246-247).

Sur le continent, certains palenques, campements où se regroupaient les marrons, mirent en péril l'existence même de la présence espagnole, en particulier au Panama actuel, où, entre 
1573 et 1577, les marrons s'allièrent aux Anglais, lesquels aspiraient, grâce à leur aide, à s'emparer des convois d'or, d'argent, de pierres précieuses qui traversaient l'isthme du port de Panamá à Nombre de Dios, sur la mer Caraïbe, où les galions de la flotte espagnole prenaient le relais jusqu'à Séville (Tardieu 2009 : 107-182). Le premier de leurs chefs, Bayano, fonda un véritable royaume. Au Mexique, sur la route de la capitale au port de Veracruz, les marrons assaillaient les convois de marchandises ou d'argent, menaçant gravement l'économie coloniale. Au début du XVII ${ }^{\mathrm{e}}$ siècle, leur chef Yanga donna beaucoup de fil à retordre au vice-roi (Pérez de Rivas, Alegre).

Jusqu'à présent, beaucoup de lieux communs ont été véhiculés sur ces deux personnages. Dans les lignes suivantes nous proposerons quelques pistes pour étudier l'influence du substrat africain sur leur comportement, plus particulièrement dans les domaines de l'organisation sociale de leurs royaumes, de la religion et de la guerre, à partir des chroniques de l'époque et des documents d'archives.

\section{1-Le royaume de Bayano}

\section{1-1-L'organisation}

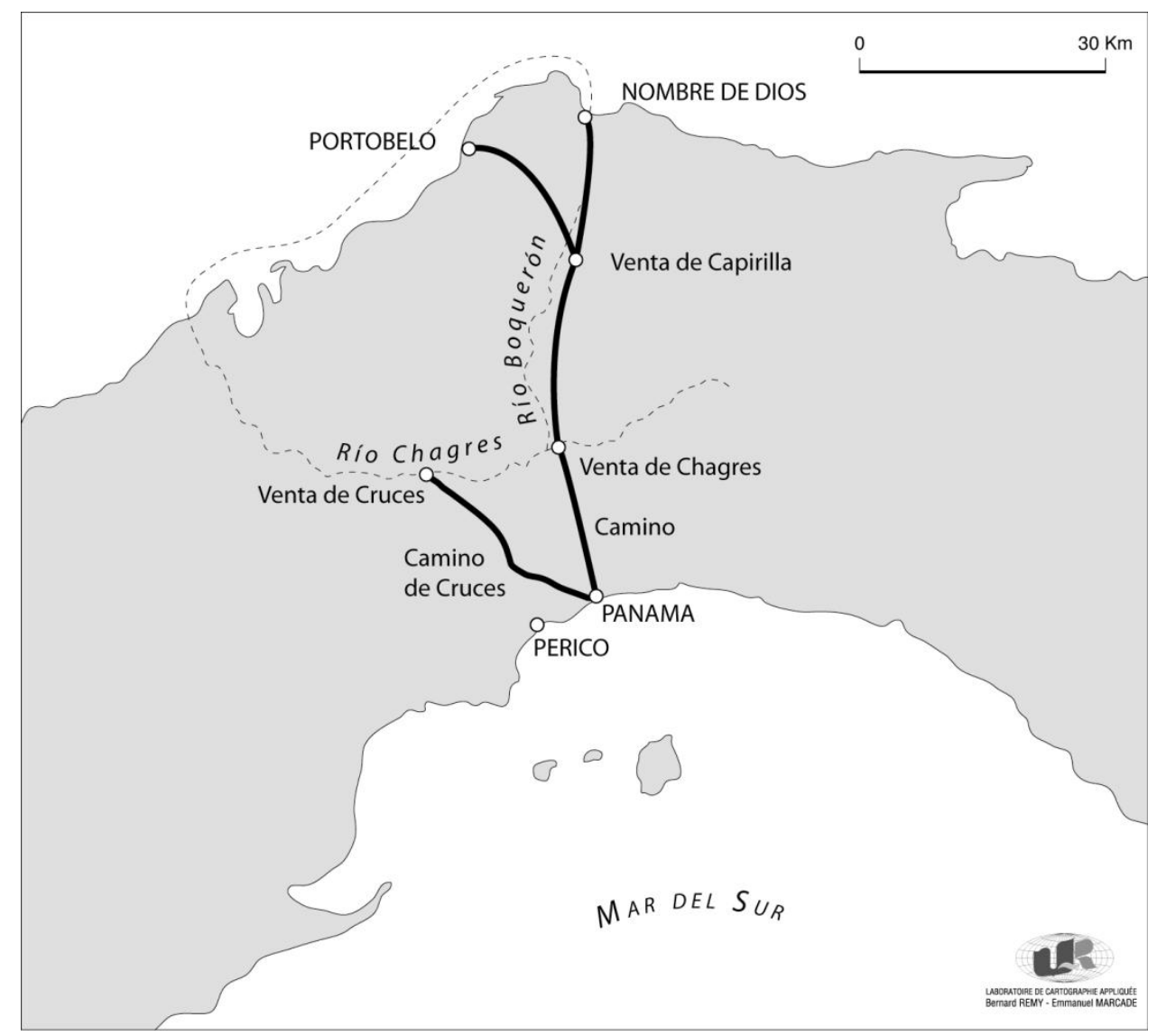

Schéma 1-Chemin de Panama à Nombre de Dios (carte établie par l'auteur)

Chez les anciens Yorubas, dans l'actuel Nigeria, Bayanni était le grand frère de Chango, le quatrième roi légendaire d'Oyo, qui avait le pouvoir de faire tomber le tonnerre selon sa volonté. Comme Chango, Bayanni, étant donné sa bonté et son élégance, devint un orisha, 
c'est-à-dire un dieu intermédiaire (Falcon 1970 : 30). En langue yoruba, «bayanni » signifie «idole » ou «objet vénéré pour les fidèles du dieu tonnerre » (Angenot et al. 1974 : 17). Cette étymologie cadre parfaitement avec notre personnage, dont le pouvoir, nous allons le voir, était à la fois politique et religieux.

Bayano (A. G. I., Audiencia de Panamá, Aguado 1919) sut se détacher de ses semblables non seulement par ses capacités militaires qui lui permirent de préserver l'indépendance des fugitifs réunis autour de lui mais aussi par ses qualités de justicier, d'homme de gouvernement. Cette personnalité correspond à l'image traditionnelle du roi africain, qui suscite le respect de ses sujets par ces capacités et ces qualités qui lui confèrent une dimension mythique. C'est ce que l'on déduira en se référant aux sermons du chef religieux de la communauté :

«Ce dont il traitait ou parlait dans ses sermons et ses prédications, c'était de persuader ses auditeurs de conserver avec obstination leur liberté en défendant les armes à la main le village et le pays qu'ils avaient et possédaient, et de soutenir leur Roi, qui s'appelait Bayamo, que tous respectaient et honoraient avec la déférence et l'obéissance dues au seigneur et roi naturel, et de la même façon que les autres gens ont l'habitude de le faire, car il devait les entretenir et les gouverner avec justice et les défendre des Espagnols qui désiraient les détruire. » (Aguado 1919 [1573-1585] : 197) ${ }^{1}$

Il le traitait de «seigneur et maître naturel », expression qui pourrait bien laisser entendre que Bayano appartenait à une famille royale africaine et qu'il aurait été victime des rivalités claniques dont tiraient profit les négriers. Il avait à cœur la défense de ce que nous oserons qualifier de « sentiment national », fondé non sur une ethnie et sur une histoire commune mais sur un terroir partagé, synonyme de liberté et de dignité, obstinément défendu contre les agressions de l'ennemi esclavagiste, c'est-à-dire sur une communauté d'intérêts ou plutôt de valeurs. Bayano dirigeait une nouvelle structure politique qui dépassait les divisions tribales africaines, comme cela apparaît clairement dans les rapports fournis à la Couronne dont disposent les Archives Générales des Indes. Elle rassemblait en effet des gens en provenance de l'Afrique de l'Ouest: "Jolofes », «Mandingas », «Branes », «Sapés », " Cazangas », Minas, Araras, Yoroubas, Congos, Angolas, mais aussi de l'Afrique de l'Est comme les Mozambiques, et des Noirs créoles.

Le siège de son gouvernement se trouvait dans un véritable « oppidum » à quelque $120 \mathrm{~km}$ de Nombre de Dios. Cette forteresse naturelle était située au sommet d'un pic en forme d'arête, entouré de profonds ravins. Il était très facile de défendre les deux étroits sentiers qui $\mathrm{y}$ donnaient accès. Les entrées du village étaient protégées par d'épaisses palissades (palenques), système possiblement hérité d'Afrique ${ }^{2}$. Entre les cases, on avait creusé de

\footnotetext{
${ }^{1}$ « Lo que en los sermones e predicaziones trataua o decia, hera persuadir a los oyentes que conseruasen con obstinazion su libertad, defendiendo con las armas en las manos el pueblo y tierra que tenían y poseían, y que sustentasen a su Rrey, que se dezia Bayamo, a quien todos acatauan y rreuerenciauan con la rreuerencia y ouidiencia que al señor y rrey natural se deue, y de la propia suerte que las otras jentes lo suelen hazer, pues los auia de mantener y gouernar en justicia y defendellos de los españoles que los deseaban destruir. »

${ }^{2}$ On peut effectivement comparer le système défensif du palenque avec celui de Mapongo, où se trouvait la capitale du roi d'Angola, selon la description de Dapper 1686 : 370 : «Le Roi d'Angole demeure au dessus de Massingan, dans un village situé sur une roche nommée Mapongo, qui a plus de dix lieuës de circuit, et dont le sommet semble atteindre jusques aux nuës. Le pié de ce côtau est bordé de plaines fertiles, baignées de ruisseaux d'eau douce, et on y trouve de tout ce qui est nécessaire à la vie. Ce rocher n'est ouvert que d'un côté et
} 
profondes cavités pour emmagasiner les vivres. Le lieu n'avait cependant qu'un usage gouvernemental et militaire. Les femmes, les enfants et les vieux vivaient en d'autres villages que ne réussirent pas à découvrir les Espagnols avant de vaincre Bayano.

Bayano n'intervenait jamais personnellement dans les opérations guerrières, qu'il confiait à ses capitaines. Sa garde le protégeait en permanence, rehaussant l'importance de son rôle. La majorité de ses sujets vivaient dans des estancias, villages satellites où se pratiquaient les cultures nécessaires au maintien de la communauté. Certains étaient à de nombreux jours de marche de la forteresse-capitale. Leur éloignement l'un de l'autre protégeait les potentialités productives de l'ensemble. En cas de besoin, chacun pouvait essaimer d'autres cellules de survie. Elles s'implantaient probablement en des lieux fertiles qui se transformaient en de nouveaux centres de colonisation agricole, capables d'assurer une production vivrière diversifiée. Etant donné les distances, ces estancias devaient jouir d'une administration propre, et d'un minimum d'organisation défensive. Tout cela relevait donc d'une vision prospective de développement agricole, politique et militaire.

inaccessible par tout ailleurs : de sorte que ce Prince n'a rien à craindre ni de la Reine Xinga, ni des Jagos ». 


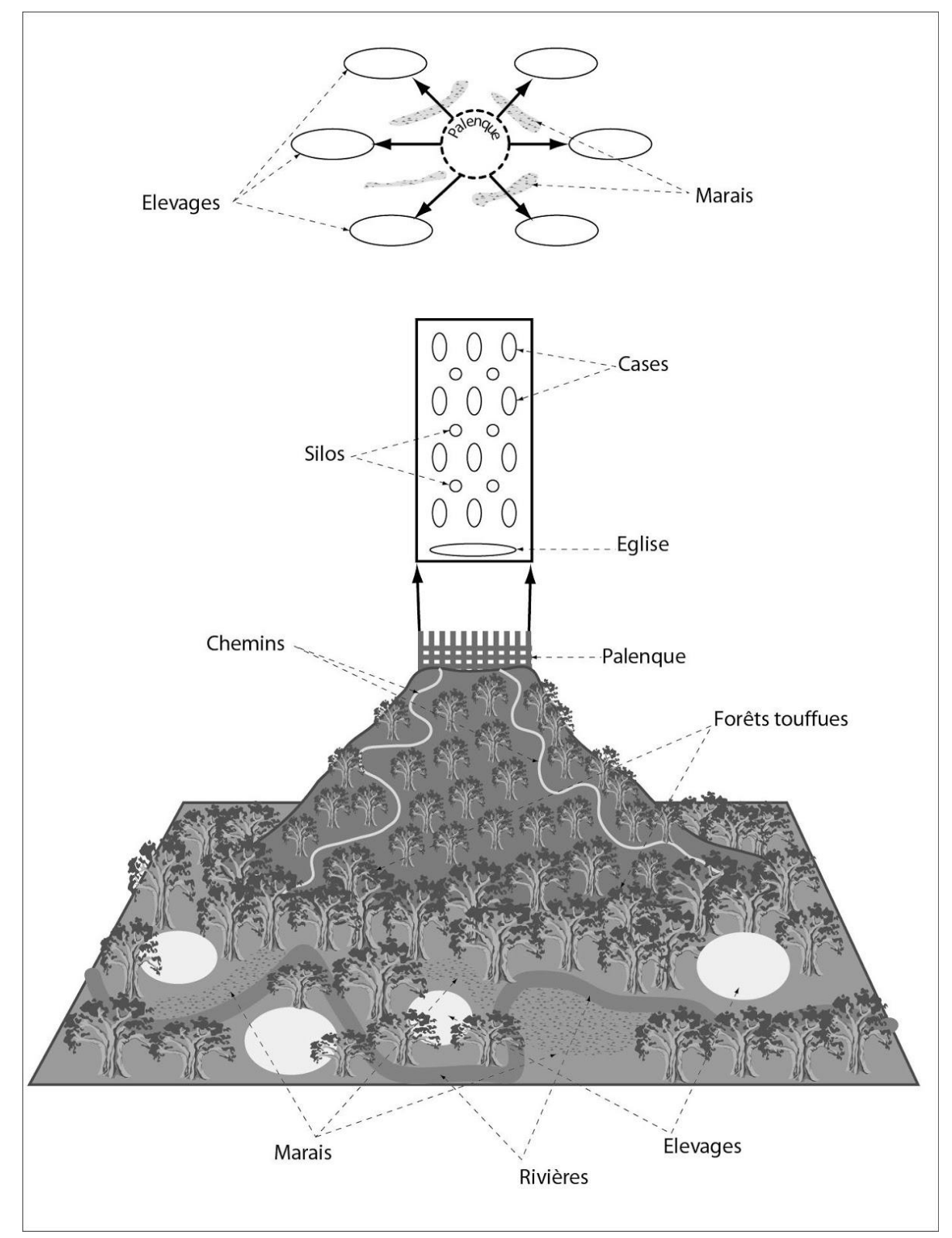

Schéma 2-Schéma de la cité-état (palenque) de Bayano

(Croquis effectué par l'auteur)

\section{1-2-La religion}

L'ébauche de sentiment national qui surgissait dans le territoire gouverné par Bayano s'inscrivait dans un contexte religieux particulier. Apparemment, il ne se produisit pas de rejet complet de la religion des anciens maîtres, étant donné le pouvoir de l'aliénation, et, peut-être, la variété du substrat africain en la matière. Le royaume se forgea une religion d'Etat, imitant le catholicisme enseigné par l'ancienne société dominante, mais avec des aspects qui dénotent un possible héritage africain. Elle renforçait l'adhésion des sujets envers leur roi, qui acquit de la sorte une légitimité de type divin. Ce serait là le «credo » de la religion nationale qui adopta par mimétisme les rites et la liturgie chrétiennes, mais en oubliant leur signification, du moins si nous nous en tenons aux affirmations du chroniqueur frère Pedro Aguado. 
Au principal responsable religieux, le «prélat spirituel », les marrons décernèrent le titre d' "évêque ». Il présidait les cérémonies revêtu de la camisole d'une femme et d'une tunique grenat. Nul doute que la liturgie catholique, élément compensatoire d'importance dans la misérable existence des esclaves, les avait profondément marqués, au point qu'ils s'en servirent pour leur affirmation identitaire. Ce personnage œuvrait dans un « sanctuaire », sorte d'église située au sommet de la montagne où se trouvait le camp.

Les rites ne se différenciaient apparemment pas de ceux pratiqués par les anciens esclaves sous la domination des Espagnols. L' «évêque » baptisait ses ouailles et leur disait la messe, en se servant d'une cruche de vin et d'un pain, entonnant des chants dans «leur langue maternelle » auxquels ils répondaient, puis il mangeait le pain et buvait le vin. Ainsi se terminait la messe, à laquelle participaient les marrons avec beaucoup de dévotion :

«...là [dans le sanctuaire], en présence de tous les assistants qui allaient l'écouter et le voir, ils mettaient une petite cruche de vin et une bon petit pain qu'ils avaient fait, et il entonnait un certain chant dans leur langue maternelle auquel répondaient les autres qui l'écoutaient et là, en présence de tous, il mangeait le pain et buvait le vin, et après cela, c'est-à-dire après qu'il eût mangé le pain et bu le vin, l'office prenait fin et tous en étaient satisfaits, et tout cela se faisait et s'écoutait avec la plus grande attention et dévotion. » (Aguado1919:197) ${ }^{3}$

La référence à la «langue maternelle » dont usait l' «évêque » pendant la messe attire l'attention. Quelle langue «vernaculaire» s'était substituée au latin, devenant ainsi expression sacrée ? Les fidèles la comprenaient-ils ou, du moins, lui avaient-ils dévolu un tel rôle ? Cela n'aurait rien d'étrange, les Africains étant habitués de tout temps à manier plusieurs langues.

L'évocation par le père Aguado du baptême pratiqué chez les marrons ne manque pas d'intérêt pour notre propos. Accompagné de danses et de libations, il était prétexte à des festivités d'indéniable origine africaine. L' "évêque », après avoir chanté et dansé comme ses fidèles, versait une cruche d'eau sur la tête de l'enfant. Puis tout le monde reprenait chants et danses et s'adonnait à la beuverie :

«Pour le baptême des nouveaux nés, ils procédaient ainsi : une fois réunis et rassemblés beaucoup de Noirs et de Noires comme parrains et marraines, ils se dirigeaient tous ensemble avec le nouveau-né vers le sanctuaire, et ils apportaient là tout le vin qu'il était possible d'avoir, ils en buvaient tous, dansaient et chantaient, ce que l'évêque lui-même faisait, et une fois cela fait il prenait une cruche d'eau et la versait sur le nouveau-né, puis tous se mettaient de nouveau à danser, chanter et boire, et ainsi se trouvait fait tout ce qu'il fallait faire et ils s'en retournaient chez les parents du nouveau baptisé. » (Aguado $1919: 197-198)^{4}$

Une fois pris, ledit «évêque » se refusa à reconnaître le catholicisme comme unique et véritable religion. Au moment de subir l'ultime châtiment, non seulement il repoussa avec

\footnotetext{
3 «...alli [en el santuario], en presencia de todos los circunstantes que le yban a huir [por "oir"] y a uer, ponian un jarraco de bino y un buen bollo de pan que hellos tenian, y cantando cierto cantar en su lengua materna, le rrespondian los demas que le estauan oyendo y alli, en presencia de todos, se comia el pan y uibia el bino, y con esto e con comer el pan y beuerse el uino acauaua su oficio y quedauan todos satisfechos, lo qual se hazia y oya con mucha atencion y devoción. »

4 «En el bautizar las criaturas tenian esta horden : que juntandose y congregandose muchos negros y negras para conpadres y comadres, se iuan todos juntos con la criatura al santuario, y alli lleuauan el bino que podia, donde uibian todos y barlauan [por "bailaban"] y cantauan, lo qual asi mesmo hazia el ouispo, y hecho esto tomaua vn jarro de agua, echauasela encima a la criatura y tornauan todos a bailar y a cantar y a ueuer, y con esto quedaua echo todo lo que auia de hazer, y se boluian a casa de los padres del rrecin [por "recién"] bautizado... »
} 
hauteur la confession et la possibilité de recevoir l'absolution, mais, empli d'une fureur démoniaque selon ses bourreaux, il affirma qu'il souhaitait la mort. Elle permettrait à son esprit de retourner dans son pays pour y recruter un grand nombre de gens afin de détruire la ville :

«...le barbare répondit, en faisant des gestes de possédé, qu'il souhaitait être déjà mort, car grâce à sa mort et à celle de ses compagnons il ferait en sorte de se venger pleinement des gens de cette ville, car après être retournés en esprits dans leur pays, ils ramèneraient un grand nombre de gens avec lesquels ils dévasteraient et détruiraient entièrement la ville. En conséquence, il ne pensait pas s'écarter de la religion que lui et les siens avaient adoptée, et dans laquelle ils entendaient bien vivre et mourir. » (Aguado $1919: 195-196)^{5}$

Bien entendu, les témoins de la scène ne saisirent pas le sens de ces imprécations, qui leur parurent sacrilèges. Elles étaient cependant à rattacher aux croyances africaines, pour lesquelles la mort n'est pas rupture, mais passage vers une autre existence, où les défunts jouissent d'une nouvelle vie leur permettant de participer avec plus de force à celle des vivants. Cela renvoie à l'ancestrolatrie, dénominateur commun de bon nombre de religions africaines (Thomas 1982). De sorte que l'aliénation, qui se manifestait chez les marrons de Bayano par les pratiques rituelles et la liturgie, n'avait pas réussi à effacer toute réminiscence du culte des ancêtres. Et le baptême, dispensé par l' "évêque », ne serait-il pas à rapprocher de la cérémonie finale de l'initiation en tant que passage à l'âge adulte - cérémonie également commune à beaucoup de religions africaines -, comme le laisseraient entendre les danses et les libations auxquelles il donnait lieu? (Baumann et Westermann 1970 : 505-507)

A bien y penser, cet étrange christianisme, né sinon d'un processus syncrétique du moins d'un effet mosaïque, correspondrait pour cette communauté afro-américaine à un besoin de construction identitaire propre. L'on comprendrait ainsi l'opiniâtreté de l' «évêque » et de ses fidèles qui moururent en martyrs de la foi qu'ils venaient d'élaborer, ciment de leur sentiment national. Plutôt que de la renier, ils préférèrent être livrés aux chiens, puis, réduits en lambeaux de chair humaine, être pendus haut et court :

«Les autres Noirs firent la même réponse que leur évêque, et ainsi les bourreaux lâchèrent quelques mâtins, des chiens de forte corpulence dont ils disposaient à cet effet, lesquels, préparés et dressés à mordre les chairs des hommes, au moment où ils les lâchèrent foncèrent sur les Noirs et commencèrent à les mordre et à les déchiqueter. Et comme les Noirs avaient en mains quelques fines baguettes pour se défendre ou menacer les chiens sans pouvoir leur faire aucun mal, cela revenait à rendre les mâtins plus furieux et à les exciter, et ainsi ces animaux, déjà plus irascibles que tout autre, faisant usage de leurs dents avec la plus grande rage, s'accrochaient aux chairs de ces pauvres Noirs, dont ils arrachaient de grands morceaux de toute part. Bien que, lors de cette agonie et ce supplice mortel, on tentât de persuader les Noirs de revenir à la foi chrétienne, ils ne voulurent jamais le faire, et ainsi, une fois bien mordus et déchirés par les chiens, on les enleva des chaînes et on les amena à un gibet monté un peu à l'écart de la ville, et là on les pendit. Ils finirent ainsi de payer le châtiment qu'ils méritaient justement de recevoir pour leur soulèvement et trahison. » (Aguado 1919: 196) ${ }^{6}$

5 «...respondio el baruaro con señales de animo endemoniado, que ya deseaua estar muerto, porque con su
muerte y la de sus compañeros pretendia auer entera benganza de la jente de aquel pueblo, porque yendo en
espiritu a su tierra trairian copia de jente con que de todo punto destruirian y asolarian la ciudad, por lo qual no
pensaua apartarse de la rreligion quel y los suyos tenian, sino hen ella entendian biuir e morir.»
6 « Los demas negros dieron la misma rrespuesta que su ouispo, y asi los uerdugos soltaron
ciertos mastines, perros de crescidos cuerpos que a punto tenian para este efeto, los quales,
como ya los tuuiesen diestros y enseñados en morder carnes de honbres, al momento que los 
Pour efficace que fût leur organisation politique et militaire, les marrons de Bayano étaient persuadés que leur royaume ne perdurerait point sans fondations religieuses originales. Nous comprendrons ainsi la raison de l'exil de leur roi en Espagne. Il avait réussi à concrétiser une utopie particulièrement dangereuse pour la paix coloniale.

\section{1-3-La guerre}

Le vice-roi Mendoza prit les choses en main en 1555 lors de son passage à Panama, en nommant comme chef de la répression Pedro de Ursúa, qui s'illustra par la suite dans l'entreprise du Marañón, en Amazonie, pour laquelle il recruta Lope de Aguirre, son futur assassin.

Les marrons eurent recours -il ne nous paraît pas impropre de l'avancer- à une polémologie particulière qui tirait sa force de leur faiblesse. Elle fit de l'écosystème leur meilleur allié pour affaiblir leurs poursuivants, dépourvus des capacités nécessaires, innées ou acquises, afin de vaincre les obstacles naturels. A cela s'ajouta le conditionnement de l'adversaire, typique de ce que serait ensuite la guerre de guérilla. Les marrons choisissaient les moments d'affrontement, lorsque l'ennemi avait le moral très bas, étant donné la fatigue, la faim et la peur. Dans «ces montagnes obscures », couverte d'une dense forêt, il ne savait jamais quand et où surgiraient les Noirs, d'où l'absolue nécessité de maintenir son attention et de se priver du sommeil réparateur. Le harcèlement incessant l'empêchait de recouvrer ses forces, ce qui accentuait son affaiblissement. Il lui était ainsi impossible de poursuivre les marrons, excellents connaisseurs des lieux où ils se mouvaient avec une extrême agilité.

La stratégie militaire céda le pas à la ruse machiavélique. Ursúa feignit d'admettre les revendications de Bayano comme seigneur de la terre. Afin d'endormir en lui toute méfiance, des rencontres amicales furent organisées entre les deux camps. Bayano, accompagné de quarante de ses meilleurs hommes, accepta même de se rendre à un banquet, où la plupart de ces derniers furent empoisonnés puis massacrés. Le chef ne put échapper au piège et la citadelle, privée de son chef charismatique et de ses lieutenants, ne tarda guère à tomber aux mains des Espagnols. C'en était fini du premier royaume marron de Panama.

\section{2-Le royaume de Yanga}

\section{2-1-L'organisation}

soltaron arremetieron a los negros y los comenzaron a morder y hazer pedazos, y como los negros tenian en las manos vnas delgadas varillas con que se defendian o amenazauan a los perros sin poder con ellas hazelles ningun daño, herales esto ocasion de henzerder e yndinar mas los mastines, y asi este animal, yracundo mas que otro ninguno, con grandisima rrauia echauan mano con los dientes y presas de las carnes destos miseros negros, de las quales arrancauan grandes pedazos por todas partes, y aunque en estas agonias y trauajos de muerte heran persuadidos los negros a que se rredujesen a la ffee, xamas lo quisieron hazer, y asi despues de bien desgarrados y mordidos de los perros, fueron quitados de las colleras y lleuados a una horca que algo apartada del pueblo tenian echa, y alli los aorcaron, con que acauaron de pagar la pena que justamente merecian rresceuir por su alzamiento y traición. » 


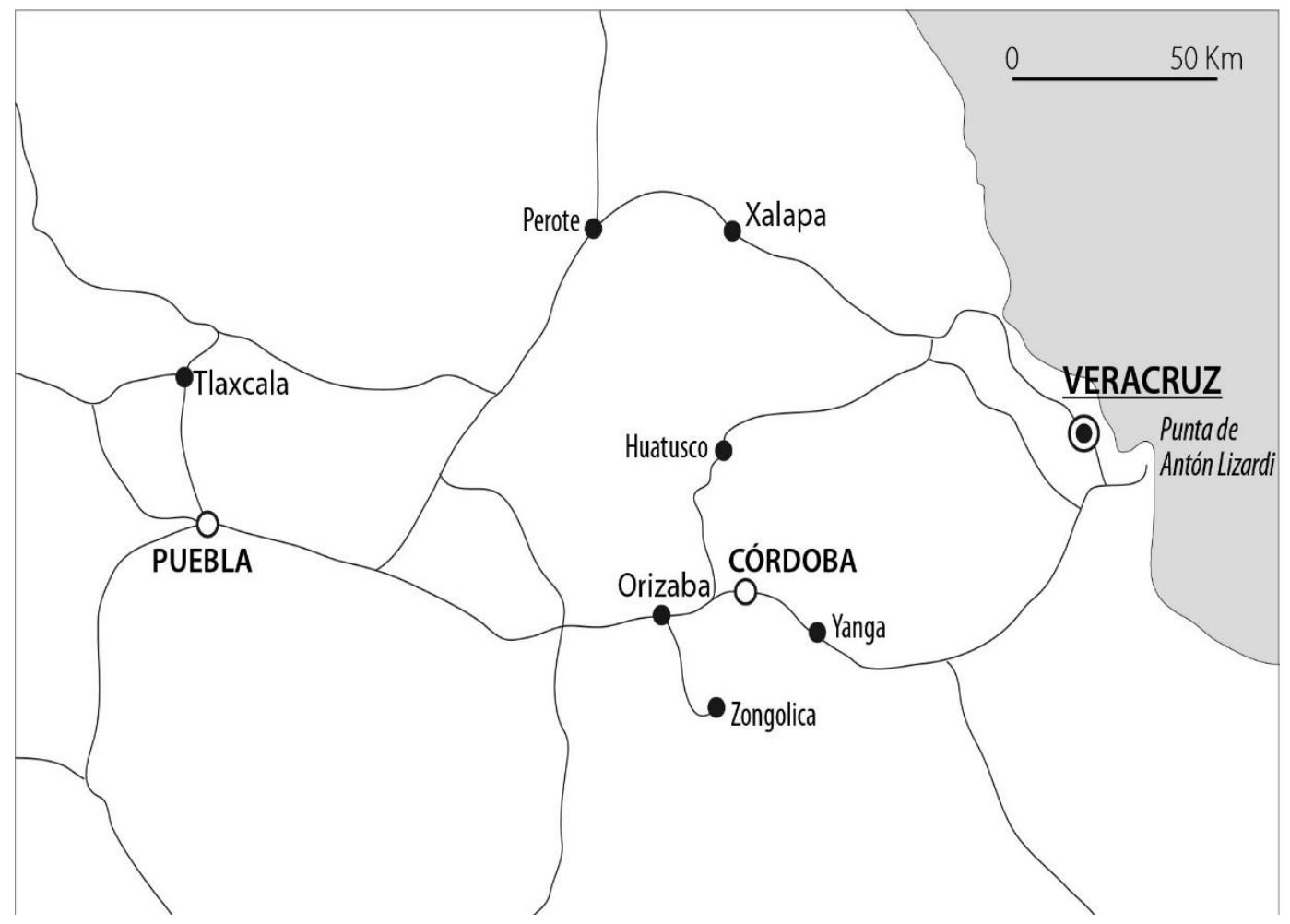

Schéma 3-Lieux du marronnage-Chemin de Mexico à Veracruz (Carte établie par l'auteur)

Depuis plusieurs décennies, on a beaucoup écrit au sujet de la résistance de Yanga dans les montagnes de l'arrière-pays de Veracruz, autour du chemin menant de la capitale de la viceroyauté au port de la Caraïbe (Tardieu 2016, à paraitre). Les informations ont été tirées principalement de la relation du père Juan Laurencio à Rodrigo de Cabredo, visiteur de la province des jésuites. Le religieux avait été nommé aumônier militaire de l'expédition de répression dirigée par Pedro González de Herrera (Pérez de Rivas 1896 : 282-294).

Selon Laurencio, en 1609, Yanga dirigeait son royaume "avec des pensées élevées » depuis trente ans, ce qui ferait remonter le début de son règne à l'an 1579. Sa réputation avait attiré de nombreux fugitifs, désireux de se placer sous sa protection et son autorité. Il sut doter son peuple d'une organisation structurée et adaptée, se réservant, avec l'âge, l'administration civile. Il confia alors le pouvoir militaire à un noir «angola », Francisco de la Matosa, peutêtre un des Jagas évoqués ci-dessous.

Le palenque principal était protégé par l'aspérité de la montagne et l'existence de marais dans le piémont. Il se trouvait précisément dans les parages de Cofre de Perote, dans les monts d'Olmealca appartenant à la sierra de Zongolica. Il dominait une campagne composée de bons pâturages, de champs de maïs, de courges et de tabac. Seul un étroit sentier conduisait au village fortifié par une muraille de roches. Ce sentier était coupé à son commencement par un long madrier servant de pont sur un cours d'eau, puis par trois portes attachées par d'épaisses lianes, et en partie obstrué de troncs d'arbres et de broussaille. De chaque côté se dressaient de hautes palissades rendant impossible une attaque massive. Du haut d'énormes rochers, les défenseurs de la citadelle pouvaient envoyer des flèches, jeter des pierres et des troncs d'arbres contre d'éventuels agresseurs obligés de progresser individuellement (Pérez de Rivas $1896: 288$ ).

La configuration du site permettait en outre aux défenseurs un repli stratégique d'un niveau à un autre, tout en continuant le harcèlement contre l'envahisseur. On remarquera la grande ressemblance de ce palenque avec celui de Bayano. 
Il se composait de soixante maisons et d'une église. Au centre se dressait un arbre très haut servant de tour de guet. A son pied se trouvait la demeure de Yanga. Dans une salle, la présence de nombreux sièges et bancs laissait penser que s'y déroulaient les réunions pour les affaires civiles et militaires. Il s'agissait donc d'une sorte de sénat, ce qui prouverait que Yanga ne régnait pas en despote (Pérez de Rivas 1896 : 288).

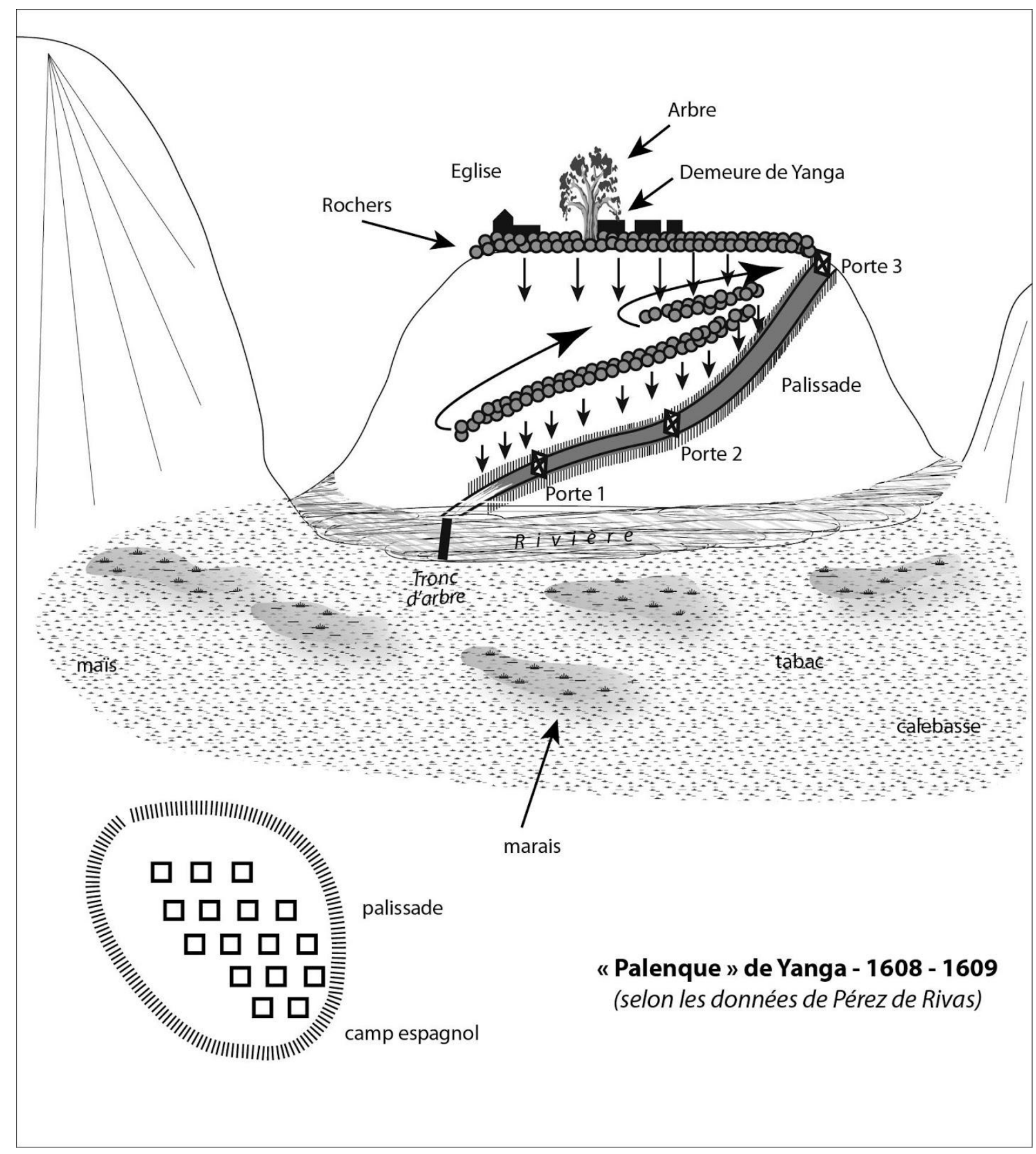

Schéma 4-Croquis effectué par l'auteur

La description du village et des champs situés au pied du pic est très significative. Une bonne adaptation au milieu écologique permettait d'abord une agriculture et un élevage différenciés, d'un grand profit pour l'équilibre de la diète marronne, et ensuite l'existence 
d'un processus de transformation des produits avec la présence d'un artisanat textile. La répartition des tâches, de par son aspect traditionnel, met en évidence la structuration de cette société, que favorisèrent la stabilité et la densité démographique. A la différence de ce qui se passait dans de nombreuses civilisations africaines traditionnelles, les hommes n'y étaient pas à la fois paysans et guerriers, mais ils se spécialisaient selon les besoins et peut-être leurs aptitudes, ce qui laisse supposer l'existence d'une sélection bien pensée, révélatrice d'une gestion prospective. La preuve en furent les abondants stocks de vivres et de produits manufacturés que trouvèrent les Espagnols, destinés à prévoir les aléas climatiques et les états de siège. Les armes, épées, fusils, provenaient du pillage ou de trafics clandestins. Les troupeaux et les chevaux avaient probablement la même origine, de même que des sommes d'argent non négligeables qui devaient permettre un certain commerce. Ainsi le royaume de Yanga, à en juger par la documentation fournie par les chroniques, ne se condamnait pas à l'autarcie ni à une simple économie parasitaire.

\section{2-2-La religion}

Le royaume de Yanga n'aurait pas rejeté ouvertement la religion des anciens maîtres, à en juger par l'existence d'une église dans la citadelle, comme dans le palenque de Bayano. Quand l'attaque se produisit, le roi marron priait dans ce sanctuaire, avec des femmes noires et indiennes, dont le rapt permettait de favoriser la progression démographique. Mais il convient de se demander s'il n'y avait pas dans les pratiques religieuses de ses sujets quelque hétérogénéité provenant du substrat africain, hypothèse à laquelle incite la rapide référence de Laurencio à la présence de flèches fichées en terre devant l'autel.

Etait-ce vraiment des flèches? Ne serait-ce pas plutôt des objets métalliques y ressemblant? Par exemple des symboles des ancêtres, appelés asê chez les peuples ewe, comme les Fons ou les Gouns de l'ancien Dahomey? Certes, ce royaume était éloigné de l'Angola, et il n'est pas du tout sûr que Yanga appartenait aux ashanti, groupe ethnique voisin des adja qui fondèrent les premiers royaumes constitutifs par la suite du Dahomey. Dans ce même espace géographique, on enterrait des barres de fer dans les sanctuaires d'Ogû, dieu des forgerons, de la chasse et de la guerre (Parrinder 1950). Si Yanga était bantou, quelques-uns de ses sujets devaient être originaires de ces contrées. Leur chef se serait donc réfugié dans l'église non point tant pour prier le dieu des chrétiens que pour solliciter la protection des ancêtres. Il aurait donc agi alors comme prêtre et les femmes présentes à ses côtés seraient des médiums chargés de transmettre les messages des hommes aux dieux. L'arbre dominant le palenque, assimilé par les Espagnols à une tour de guet, aurait bien pu être un arbre sacré, en relation avec le culte des ancêtres, selon les usages de la Côte des Esclaves, en Afrique occidentale (Juhé-Beaulaton 1999 : 102).

Mais si, en ces lieux, le forgeron qui travaillait le fer (hountondji) jouait un rôle religieux transcendantal, il en était de même dans l'ancien royaume du Congo, en particulier lors de la prise de pouvoir des souverains. Dans cette aire, signale De Heusch 2000 : 106-112, les chefs maniaient traditionnellement des marteaux de forge (nzundo), donnant à voir que, comme le forgeron, ils étaient responsables de la prospérité générale ${ }^{7}$.

Certes, il serait quelque peu hasardeux de comparer des marteaux à des flèches. On retiendrait donc le lien intime entre la royauté et la forge, aspect commun entre les deux aires africaines, dont le substrat se serait manifesté dans l'église du palenque de Yanga.

${ }^{7}$ L'anthropologue emprunte la référence au couronnement de Pedro II à Jadin, L, 1975, L'ancien Congo et l'Angola (1639-1655), d'après les sources romaines, portugaises, néerlandaises et espagnoles, Bruxelles. 
Mais il y a plus. Si nous en croyons Laurencio, le comportement des marrons pendant leurs expéditions était particulièrement barbare. Il s'attarde sur le sacrifice rituel d'un Espagnol, dont, après lui avoir ouvert la tête, ils burent le sang «avec des manifestations de superstition et des cérémonies barbares ». Si le jésuite ne se trompa guère dans la qualification de l'acte (« superstition », « cérémonies »), il n'en comprit nullement la portée.

A ce sujet, une lecture serait fort éclairante : celle de la relation d'Antonio Cavazzi de Montecuccolo, missionnaire capucin italien au Congo, au Matamba et en Angola, écrite à la demande de Propaganda Fide et publiée à Bologne en 1687. Une grande partie du texte tourne autour de la conversion de la mythique reine d'Angola, ou plutôt, de Matamba et de Ndongo, Njinga Mbandi (1582-1663), à laquelle les Portugais donnèrent, lors de son baptême, le patronyme du gouverneur de Luanda, d'où son nom de «doña Ana de Sousa ». Ce qui nous intéresse plus particulièrement dans cette œuvre, ce sont les descriptions du comportement de la secte des Jagas, dont la reine adopta les coutumes lors de ses luttes contre ses rivaux et même contre les Portugais, avant de revenir à la foi chrétienne à la fin de sa vie. Les terribles Jagas avaient l'habitude de manger leurs ennemis vaincus et de sucer leur sang.

Ce que ne pouvait indiquer Laurencio (Pérez de Rivas 1896: 45) c'est que le rite ne correspondait pas à la simple satisfaction d'un instinct animal mais bien au désir des guerriers de s'approprier les forces vitales du vaincu, coutume pratiquée par plusieurs ethnies africaines. Reste à savoir comment seraient arrivés ces rites jusqu'à nos marrons. Nous avons déjà fait allusion aux origines angola d'une bonne partie d'entre eux, à cause de la traite négrière dominée alors par les Portugais. Selon la relation de Cavazzi, un des châtiments les plus utilisés par Njinga envers ses adversaires consistait à les envoyer " outre-mer pour servir dans les mines des blancs ». Ses colères n'épargnaient pas les personnages les plus en vue, chefs locaux ou soba (Cavazzi 1687 ; trad. par de Castro et du Cheyron d'Abzac 2010 : 213, 221, 247, 249, 283, 304). Une fois transférées aux Indes occidentales, rien de surprenant donc, si l'on tient compte des défaillances ou de la superficialité de l'instruction chrétienne dispensée aux esclaves, à ce que ces victimes de la traite continuent à pratiquer ces rites lorsque les circonstances leur semblaient l'imposer.

Yanga aurait pu être un de ces esclaves, si nous nous en tenons aux dires de Laurencio (Pérez de Rivas 1896 : 8), selon lequel il appartenait à la nation «bran » et aurait pu être roi de son pays s'il n'avait été capturé. Les «Bran» correspondraient aux Abrons de l'ancienne Côte de l'or, le Ghana actuel. Mais selon Ngou-Mve (1997 : 40), cette appellation évoquerait plutôt les «Bram» ou «Brama» (de «bavarama») de l'Afrique centrale. Quoi qu'il en fût, répétons-le, nombre des sujets de Yanga provenaient du royaume de Matamba ou de Ndongo, où s'imposaient les coutumes des Jagas, et peut-être durant le règne de la reine Njinga ellemême. En ce cas, ce seraient effectivement de redoutables guerriers, ce qui expliquerait les difficultés éprouvées par González de Herrera pour dominer les « yanguicos », comme on se mit à appeler les fugitifs dirigés par Yanga (Pérez de Rivas 1896 : 285).

\section{2-3-La guerre}

Le 30 mai 1610, le vice-roi Luis de Velasco informa la Couronne de la situation. Le capitaine Pedro González de Herrera avait été chargé de mener à bien l'entreprise de répression. Il y eut de nombreux et violents affrontements qui, s'ils délogèrent les Noirs de leurs camps, ne réussirent pas à les anéantir. Comme les sujets de Bayano, les marrons de Yanga avaient adopté une organisation territoriale stratégique qui les protégeait des agressions, en se divisant en plusieurs groupes, les rancherías, qui s'administraient avec une certaine autonomie. Même sous l'effet de la torture, les prisonniers étaient dans l'incapacité de révéler l'emplacement des autres campements, seul connu des proches de Yanga. 
González de Herrera avait réuni une troupe de cinq cents hommes : cent soldats de métier, autant d'Espagnols aventuriers, cent cinquante Indiens armés d'arcs et de flèches, plus deux cents individus d'origines diverses : Espagnols, Mulâtres et Métis des estancias voisines. Selon les estimations de Laurencio, l'armée des marrons ne dépassait pas quatre-vingts hommes de combat (Pérez de Rivas 1896 : 290).

Lors d'un affrontement, ces derniers épargnèrent la vie d'un Espagnol. Amené au camp, le chef marron le traita avec magnanimité, comme un monarque oint par la divinité : « ... ne crains rien, Espagnol, lui dit-il, tu ne mourras pas, car tu as vu mon visage ». Avec le temps, Yanga se crut peut-être investi de quelque mission sacrée, de là cette attitude que ne put comprendre Laurencio, qui le traita d'orgueilleux. Cela apparaît en toute clarté dans le message confié au prisonnier pour le capitaine espagnol :

«...ils s'étaient retirés en cet endroit pour se libérer de la cruauté et de la perfidie des Espagnols qui, sans aucun droit, prétendaient être maîtres de leur liberté. Dieu favorisant une cause si juste, ils avaient, jusqu'alors, obtenu de glorieuses victoires sur tous les Espagnols qui étaient venus les appréhender. En attaquant les hameaux et les haciendas des Espagnols, ils ne faisaient que se dédommager, par la force des armes, de ce qu'on leur refusait injustement. » (Pérez de Rivas $1896: 292)^{8}$

Cette réfutation débouchait logiquement sur une justification du comportement des marrons : leurs agissements, présentés comme des excès abominables, n'étaient que défense de leur droit et compensation des malheurs imposés par l'esclavage. Et, si cela ne suffisait pas, Yanga les situait habilement dans le cadre du providentialisme chrétien : sa résistance, durant trente ans, lui paraissait comme une manifestation de la bienveillance divine. Sa rhétorique consista à se prévaloir de l'idéologie de ses ennemis en mettant le doigt dans leur contradiction. Il semblerait donc pertinent de parler de messianisme de la part du chef marron qui trouva dans la foi de la société esclavagiste les armes dialectiques nécessaires à sa justification.

Parvenus enfin au pied du sommet où se trouvait la citadelle, les Espagnols construisirent un camp fortifié. Le 23 février 1609, à trois heures du matin, le signal de l'assaut fut donné. Les assaillants reçurent une pluie de flèches, de pierres et de troncs d'arbres. Le capitaine et l'aumônier furent blessés. Les obstacles accumulés sur le sentier devenaient de plus en plus difficiles à surmonter. Mais les agressés se virent obligés d'abandonner la défense des portes. Yanga, informé des mauvaises nouvelles, adressa en vain des encouragements à ses hommes Les dernières défenses ne parvenant pas à contenir l'ennemi, le sauve-qui-peut commença vers les forêts voisines. Le lendemain, le capitaine espagnol essaya d'entrer en pourparlers avec les fuyards qui lui opposèrent une farouche résistance. Yanga avait eu le temps de se réfugier dans un autre campement d'où il organisa le harcèlement des ennemis, avant de devoir fuir de nouveau. L'affaiblissement de ses hommes le mit dans l'obligation de solliciter du vice-roi des conditions acceptables de reddition. Nous ne savons rien des tractations. Par contre nous disposons du texte des accords qui accordaient la liberté aux marrons, regroupés

${ }^{8}$ «...ellos se havían retirado a aquel lugar, por libertarse de la crueldad y de la perfidia de los españoles, que sin algún derecho, pretendían ser dueños de su libertad ; que favoreciendo Dios una causa tan justa, havían, hasta entonces, conseguido gloriosas victorias de todos los españoles que havían venido a prehenderlos. Que en asaltar los lugares y haciendas de los españoles, no hacían sino recompensarse, por fuerza de las armas, de lo que injustamente se les negaba. » 
dans une réduction proche de la citadelle. En échange, ils seraient contraints d'aider à la capture des futurs fugitifs. Ainsi fut créé le village de San Lorenzo de los negros, d'une population de trois cents habitants. Ils éliraient leurs responsables, sauf en matière de justice, confiée à un espagnol, et seraient soumis à l'enseignement religieux dispensé par un curé. Yanga fut donc le premier dirigeant du village, charge transmise après sa mort à ses descendants, lesquels durent affronter d'autres difficultés avec les autorités du bourg voisin de Córdoba, qui éprouvèrent la plus grande réticence à admettre cette autonomie et tentèrent par tous les moyens d'y porter obstacle.

Bayano et Yanga étaient donc loin d'être de simples chefs de bande, des sauvages sans aucun scrupule comme le laisse entendre la documentation référée ci-dessus. Celle-ci cependant fournit des éléments qui donnent matière à réflexion, à condition que l'on fasse le lien avec les civilisations africaines, telles qu'elles apparaissent dans les chroniques de voyageurs ou de religieux des $\mathrm{XVI}^{\mathrm{e}}$ et $\mathrm{XVII}^{\mathrm{e}}$ siècles, voire dans les analyses des anthropologues actuels. Ce que les Espagnols prenaient pour des actes de barbarie relève souvent de croyances et de pratiques authentiquement africaines, du moins dans ce qu'elles avaient de commun. Le culte des ancêtres, élément essentiel de l'identité africaine en général, se mélangea avec les croyances chrétiennes, effet de l'aliénation imposée par la société esclavagiste, pour produire une nouvelle identité qui aurait pu prospérer si ces sociétés en voie de gestation n'avaient été impitoyablement détruites par de véritables guerres menées parfois depuis l'Espagne même, comme ce fut le cas dans la province de Panamá, ou qui avaient mobilisé des forces extrêmement importantes dans les vice-royautés du Pérou ou du Mexique. 


\section{Sources manuscrites}

Archivo General de Indias (A. G. I.), Audiencia de Panamá, liasses 1, 11, 13, 14, 15, 29, 30, $39,40,51,52$.

\section{Sources imprimées}

AGUADO Pedro de, 1919 [1573-1585?], Historia de Venezuela, Edición de Jerónimo Bécker, Madrid, Establecimiento tipográfico de Jaime Ratés.

ALEGRE Francisco Javier, 1958 [1597-1639], Historia de la provincia de la Compañía de Jesús de Nueva España, Nueva edición por Ernest J. Burrus s. j. y Félix Zubillaga s. j., Roma, Institutum Historicum S. J.

CAVAZZI DA MONTECUCCOLO Giovanni Antonio, 1687, Istorica descrittione de'tre regni Congo, Matamba et Angola situavi nell'Etiopia inferiore occidentale e delle missioni apostoliche esercitatevi da religiosi capuccini, accuratamente compilada dal p. Gio. Antonio Cavazzi da Montecuccolo .... Bolonia. Nous utilisons la traduction de Xavier de Castro, Xavier de, et Alix du Cheyron d'Abzac publiée sous le titre de Njinga, reine d'Angola, Paris, Chandeigne, 2010.

DAPPER Olivier, 1686, Description de l'Afrique, Amsterdam, Wolfgang, Waesberge, Boom et van Someren.

PÉREZ DE RIVAS Andrés, 1896 [1654], Corónica y historia religiosa de la Provincia de la Compañía de Jesús de México en Nueva España, México, Imprenta del Sagrado Corazón de Jesús.

\section{Références bibliographiques}

ANGENOT Jean-Pierre, JACQUEMIN Jean-Pierre, VINCKE Jacques L., 1974, Répertoire des Vocables Brésiliens d'origine africaine, Université Nationale du Zaïre, Faculté des Lettres, Centre de Linguistique Théorique et Appliquée.

BAUMANN H. et WESTERMANN, D, 1970, Les peuples et les civilisations de l'Afrique, Paris, Payot.

FALCON Paul, 1970, «Religion du vodun », Etudes Dahoméennes 18-19, Cotonou, Institut de Recherches Appliquées du Dahomey.

HEUSCH Luc de, 2000, Le roi de Kongo et les monstres sacrés, Paris, Gallimard.

JUHÉ-BEAULATON Dominique, 1999, «Arbres et bois sacrés de l'ancienne Côte des Esclaves. Des repères historiques », in : Jean-Pierre Chrétien et Jean-Louis, 1999, Histoire d'Afrique. Les enjeux de la mémoire, Paris, Karthala : 101-118.

NGOU-MVE, Nicolas, 1997, «El cimarronaje como forma de expresión del Africa bantú en la América colonial : el ejemplo de Yangá en México », América Negra 14, Bogotá, Colombie, Pontificia Universidad Javeriana : 27-51. 
PARRINDER Geoffrey, 1950, La religion en Afrique occidentale, Paris, Payot.

TARDIEU Jean-Pierre, 2006, « Cimarrón-Marroon-Marron. Note épistémologique », OutreMers. Revue d'Histoire (350-351) : 237-247.

-2009, Cimarrones de Panamá. La forja de una identidad afroamericana. S. XVI, Frankfurt / Madrid, Vervuert / Iberoamericana.

-2016 [à paraître], Resistencia de los negros en el virreinato de México (S. XVI-XVII), Frankfurt / Madrid, Vervuert / Iberoamericana.

THOMAS Louis-Vincent, 1982, La mort africaine, Paris, Payot. 